Fernández García, F. (2020): La potenciación descortés del desacuerdo en hablantes españoles e ingleses. Cultura, Lenguaje y Representación, Vol. XXIII, 65-82

ISSN $1697-7750 \cdot$ e-ISSN 2340-4981

DOI: http://dx.doi.org/10.6035/clr.2020.23.4

\title{
La potenciación descortés del desacuerdo en hablantes españoles e ingleses
}

Impolite boosting of disagreement in Spanish and English speakers

FRANCISCO FERNÁNDEZ GARCÍA

UNIVERSIDAD DE JAÉN

Artículo recibido el / Article received: 2019-06-05

Artículo aceptado el / Article accepted: 2019-07-16

RESUMEN: En el marco de un trabajo de investigación más amplio acerca de la gestión del desacuerdo por parte de hablantes españoles e ingleses, este artículo se centra en el análisis y la caracterización del modo en que dichos hablantes llevan a cabo la potenciación descortés del desacuerdo en un entorno conversacional de tensión, estableciendo una comparación con resultados previos sobre la mitigación cortés característica de un contexto distendido. La investigación toma como marco de referencia la teoría del rapport management de Spencer-Oaety $(2002,2008)$ y analiza el comportamiento descortés en torno a un triángulo metodológico formado por las estrategias discursivas utilizadas, los mecanismos lingüístico-discursivos mediante los que tales estrategias se implementan y las repercusiones sociales de los actos lingüísticos. Los resultados demuestran que a) el uso de las estrategias de potenciación descortés sigue patrones diferenciados respecto de la mitigación cortés, b) pueden aislarse estrategias y mecanismos recurrentes en la ejecución de dicha potenciación, y c) el uso de estrategias y mecanismos define perfiles apreciablemente diferenciados entre hablantes españoles e ingleses.

Palabras clave: desacuerdo, potenciación, descortesía, pragmática intercultural, estrategias, mecanismos.

ABSTRACT: Within the framework of a broader research work on the management of disagreement by Spanish and English speakers, this paper focuses on the analysis and characterization of the way in which these speakers carry out the impolite boosting of disagreement in a conversational environment of tension, establishing a comparison with previous results on the polite mitigation frequently used in a relaxed context. The research takes as reference frame the rapport management theory of Spencer-Oaety $(2002,2008)$ and analyzes impolite behavior according to a methodological triangle formed by the discursive strategies used, the linguistic-discursive mechanisms by means of which such strategies are implemented and the social effects of linguistic acts. The results show that a) the use of impolite boosting strategies follows differentiated patterns than that of polite mitigation, b) specific strategies and mechanisms can be found that are recurrently used in this boosting, and c) the 
use of strategies and mechanisms defines appreciably differentiated profiles between Spanish and English speakers.

Key words: disagreement, boosting, impoliteness, intercultural pragmatics, strategies, mechanisms.

\section{INTRODUCCIÓN}

Las investigaciones sobre la (des)cortesía lingüística constituyen, sin duda, uno de los principales centros de interés de los estudios pragmáticos actuales. Los desarrollos teóricos y aplicados desde los planteamientos pioneros de Brown y Levinson $(1978,1987)$ han sido incontables, con propuestas dirigidas a reformular el modelo original (como Spencer-Oatey, 2002, 2008; o, en el ámbito hispánico, Bravo, 1999, 2003), en unos casos, o bien a negar su viabilidad (como Watts, 2003; o Locher y Watts, 2005), en otros; y, paralelamente, con propuestas dirigidas específicamente al análisis de la descortesía, a partir del trabajo pionero de Culpeper (1996), con trabajos de referencia como Bousfield (2008) y Culpeper (2011). Por otro lado, la investigación de la dimensión intercultural de la comunicación, y en concreto de lo relativo a la (des)cortesía lingüística, se viene configurando también en las últimas décadas como otro de los grandes polos de interés de los estudios pragmáticos, en el que se cuenta ya con obras punteras de referencia (como Wierzbicka, 2003; o Spencer-Oatey, ed., 2008). En la intersección entre ambos campos, esto es, en el estudio intercultural de la (des)cortesía, se centra la presente aportación, concretamente en el estudio del comportamiento descortés de hablantes españoles e ingleses en la formulación de desacuerdos.

En diferentes publicaciones recientes de índole teórica (Fernández García, 2016a; Fernández García, 2016b; Fernández García y Ortiz Viso, 2018) hemos reflexionado sobre la necesidad de que las investigaciones pragmáticas de carácter intercultural, en general, y particularmente las referidas a la (des)cortesía, no conciban como bloques monolíticos los entornos culturales a los que se acercan, puesto que, como han puesto ya de manifiesto no pocas investigaciones (por ejemplo, Schneider, 2012; o Staley, 2018), la variación pragmática no aparece únicamente asociada a la diferencia interlingüística, sino que también acaece dentro de una misma lengua.

Desde tal convicción pusimos en marcha nuestra investigación sobre el desacuerdo, desarrollada ya en varios trabajos (Fernández García y Aguayo Cruz, 2019; Fernández García, en evaluación; Fernández García, en prensa). Los resultados de dichos trabajos han dejado clara, entre otras cosas, la enorme presión que la variación situacional llega a ejercer sobre el comportamiento de los hablantes a la hora de configurar sus desacuerdos, hasta el punto de que ciertos contextos muestran una aparición ampliamente mayoritaria de formulaciones tendentes a la mitigación cortés, mientras que otros, de forma inversa, propician una casi sistemática tendencia a la potenciación descortés del desacuerdo.

A partir de tales hallazgos, este trabajo se centra en el estudio de un marco contextual propiciador de la potenciación descortés del desacuerdo, analizando la forma en que este se configura y la existencia de posibles patrones culturales diferenciados al respecto. De manera más concreta, nos proponemos los siguientes objetivos: 
a) Caracterizar el modo en que se configuran las acciones de potenciación del desacuerdo, contraponiéndolo a la caracterización previa de aquellos otros casos en los que lo que se pretende es mitigar su fuerza asertiva.

b) Distinguir, en la caracterización anterior, las estrategias funcionales utilizadas por los hablantes y los mecanismos lingüístico-discursivos mediante los que las implementan.

c) Llevar a cabo los objetivos a) y b) desde una perspectiva intercultural, aislando rasgos caracterizadores de los respectivos comportamientos de los hablantes españoles y los ingleses.

\section{MARCO TEÓRICO-METODOLÓGICO Y MUESTRA DE ANÁLISIS}

El enfoque teórico desde el que abordamos el análisis de la (des)cortesía es la teoría del rapport management, desarrollada por Spencer-Oatey a lo largo de distintas publicaciones (como 2002, 2005, 2007 y 2008). Conforme a ella, distinguimos dos aspectos esenciales en la proyección social del individuo, a saber, su imagen y sus derechos de socialización. La primera comprende, a su vez, la imagen cualitativa, la imagen social y la imagen relacional; y los segundos, por su parte, los derechos de equidad y los derechos afiliativos. Consideramos que la propuesta de Spencer-Oatey constituye un desarrollo que corrige y hace más potente el modelo clásico de Brown y Levinson $(1978,1987)$, frente a los planteamientos teóricos que surgen como un rechazo a este, pero que realmente no ofrecen una alternativa productiva desde un punto de vista práctico.

Dicho marco teórico se engarza en nuestro trabajo con un planteamiento metodológico, conforme al cual, el funcionamiento de lo (des)cortés es entendido como una conjunción de estrategias y mecanismos cuyo uso lleva aparejadas determinadas repercusiones sociales (Fernández García, 2016b: 88-91). Las estrategias funcionales definen el propósito discursivo del hablante y poseen, per se, un determinado perfil social, es decir, están orientadas a la consecución de un determinado efecto; los mecanismos lingüístico-discursivos son el canal de ejecución de tal propósito funcional, es decir, un mero soporte; las repercusiones sociales de los actos, por último, vienen condicionadas por el propósito funcional de la estrategia utilizada por el hablante y tienen que ver con las consecuencias del acto lingüístico en relación con los diferentes planos de la proyección social del individuo (la imagen y los derechos de socialización, con sus subtipos).

En lo tocante a la muestra de análisis, partimos de la información obtenida mediante un cuestionario que, en sendas versiones española e inglesa, fue suministrado en 2016 a informantes de los entornos de las ciudades de Jaén (Andalucía, España) y Coventry (Midlands Occidentales, Inglaterra). Trabajamos sobre 240 informantes, 120 de cada una de las procedencias, seleccionados conforme a tres variables sociales, a saber, sexo, edad y formación académica. El cuestionario consta de diez preguntas, con las que busca obtener información relativa a distintos aspectos concernientes a la percepción y gestión de lo (des)cortés por parte de los hablantes. Dos de tales preguntas, la 3 y la 4 , están relacionadas específicamente con el desacuerdo y son las que nos interesan aquí. Las presentamos en su versión española:

3. Imagina la siguiente situación. Te encuentras charlando animadamente, en un ambiente distendido y de confianza, con un/a amigo/a (compañero/a de trabajo, vecino/a, etc.). Estáis en casa o en un restaurante. Hablando sobre algún asunto de actualidad que te interesa mucho, tu amigo/a expresa alguna opinión con la que no estás en absoluto de acuerdo. ¿Cómo reaccionarías? Intenta reproducir aquí cuáles podrían ser tus palabras. 
Imagina ahora que te ocurre algo parecido (no estás en absoluto de acuerdo con lo dicho por tu interlocutor), pero en la consulta del médico, con el que no tienes ninguna especial relación de confianza. ¿Qué dirías?

4. Piensa ahora que la conversación con tu amigo/a (de la pregunta anterior) se va acalorando, con una sucesión de desacuerdos, hasta convertirse en una discusión. Entonces, él/ella te lleva la contraria de una forma especialmente crítica, que te hace sentirte muy molesto/a (por ejemplo, diciéndote que dices eso porque no tienes ni idea de lo que estás hablando). ¿Cómo reaccionarías? Intenta reproducir aquí cuáles podrían ser tus palabras.

Como puede observarse, se trata de preguntas de carácter abierto, hecho que nos ha permitido combinar el análisis cualitativo con el cuantitativo: un análisis cualitativo inicial, capaz de proporcionar la riqueza de matices ofrecidos por el informante, y un análisis cuantitativo posterior a partir de las categorías analíticas obtenidas en el análisis cualitativo. En cuanto a la naturaleza concreta de las preguntas 3 y 4, vemos que en la pregunta 3 se incide en la relevancia del cambio de marco comunicativo, planteando dos alternativas, un contexto informal y de confianza frente a un contexto formal y sin confianza en el que acaece una relación social asimétrica (las llamaremos en adelante $3 \mathrm{a}$ y 3 b); la pregunta 4, por otro lado, se plantea como una continuación de la primera parte de la 3 y pone el foco en la transformación de un mismo marco comunicativo, analizando la actitud del hablante ante la desaparición de la cordialidad y el surgimiento de un ambiente hostil en el contexto informal.

\section{AVANCES PREVIOS EN LA INVESTIGACIÓN}

Señalábamos en $\S 1$ que la variación situacional llega a condicionar sustancialmente la formulación de los desacuerdos, propiciando que la mitigación o la potenciación de dicho acto de habla lleguen a ser ampliamente mayoritarias, según el caso. Concretemos ahora dicha afirmación general con los datos de la tabla 1, procedente de Fernández García (en evaluación):

Tabla 1. Porcentaje de informantes que muestran un desacuerdo mitigado, abierto o potenciado en cada contexto

\begin{tabular}{|c|c|c|c|c|c|c|c|c|c|}
\hline & \multicolumn{3}{|c|}{ Pregunta 3a } & \multicolumn{3}{|c|}{ Pregunta 3b } & \multicolumn{3}{|c|}{ Pregunta 4} \\
\hline & \begin{tabular}{|l|} 
mitigado \\
\end{tabular} & abierto & potenc. & mitigado & abierto & potenc. & mitigado & abierto & potenc. \\
\hline Jaén & $68,35 \%$ & 17,72 & $3,92 \%$ & $88,10 \%$ & $7,14 \%$ & $4,76 \%$ & $11,36 \%$ & $9,09 \%$ & $79,55 \%$ \\
\hline oventry & $80,33 \%$ & $11,48 \%$ & $8,20 \%$ & $67,74 \%$ & $22,58 \%$ & $9,68 \%$ & $11,54 \%$ & $11,54 \%$ & $76,92 \%$ \\
\hline
\end{tabular}

Como puede observarse, se trata de los porcentajes de informantes que, en cada uno de los tres contextos analizados en nuestra investigación, optaron por formular su desacuerdo de una forma mitigada, abierta o potenciada. ${ }^{1}$ Sin entrar ahora en otras cuestiones, resulta manifiesto que la opción por un desacuerdo mitigado fue ampliamente mayoritaria en ambas nacionalidades en los dos contextos no conflictivos, tanto el informal de $3 \mathrm{a}$ (conversación distendida con el amigo) como el formal de $3 \mathrm{~b}$ (conversación con el médico), mientras que la preferencia por la potenciación del desacuerdo fue nítida en el contexto de tensión de 4 (conversación con el amigo, convertida en discusión). Por dicho

\footnotetext{
${ }^{1}$ Es decir, los casos en los que el hablante utiliza procedimientos para, respectivamente, suavizar o reforzar la asertividad de su enunciación (mitigada vs. potenciada), y aquellos en los que la presenta de forma neutra (abierta). En este mismo epígrafe se presentan las estrategias utilizadas para la función mitigadora; en $\S 4$ se detallan las estrategias y los mecanismos usados para la función potenciadora.
} 
motivo, para poder contar con datos representativos, nuestro análisis sobre la potenciación del desacuerdo en este trabajo se centrará en las respuestas a la pregunta 4.

Por otro lado, los resultados obtenidos en Fernández García (en evaluación) nos llevaron a cuestionarnos sobre el posible carácter descortés del desacuerdo. La idea había quedado descartada como un apriorismo, en la medida en que se han descrito diferencias culturales al respecto. Sin embargo, hemos constatado (tabla 1) que la mitigación es bastante generalizada en los contextos no conflictivos ( $3 a$ y $3 b$ ), mientras que la potenciación se generaliza en el contexto de tensión (4); y a ello debemos añadir que la formulación de desacuerdos, en general, se reduce bruscamente en dicho contexto de tensión (4) frente a las cifras de los contextos previos (particularmente de 3a). ${ }^{2}$ De todo ello parece deducirse que el desacuerdo es considerado, tanto por los españoles como por los ingleses, un acto potencialmente dañino para el equilibrio de las relaciones sociales con el interlocutor. Y esa es, probablemente, la causa de que se configure, en principio - preguntas $3 \mathrm{a}$ y $3 \mathrm{~b}$ - , como la segunda parte no preferida - tanto estructural (Levinson, 1983: 336) como psicológicamente (Bousfield, 2008: 237) — de los pares de adyacencia asertivos en que aparece. Sin embargo, cuando la conversación se tensa y acalora, y se produce la transformación estructural (Kotthoff, 1993) que acaba por convertirla en una discusión -el caso de la pregunta 4-, la preferencia cambia y el desacuerdo se convierte en la opción estructuralmente preferida, aunque siga resultando psicológicamente no preferida, discordancia de la que surge su configuración abiertamente descortés (el hablante opta por formular de manera clara, directa, concisa, etc., su desacuerdo preferencia estructural - a pesar de la conciencia de que su interlocutor prefiere preferencia psicológica - lo contrario). En definitiva, por tanto, podemos concluir que, en lo relativo a los contextos descritos, el desacuerdo se revela como un acto verbal potencialmente descortés, cuya mitigación se traduce en un efecto cortés y cuya potenciación se traduce en un efecto descortés, y que ello ocurre, a grandes rasgos, de manera semejante para los informantes de ambas nacionalidades. ${ }^{3}$

Tal es el motivo de que establezcamos en nuestro estudio una correlación entre la formulación mitigada del desacuerdo y un perfil cortés, por un lado, y su formulación potenciada y un perfil descortés, por otro. Partiendo de dicha distinción, en Fernández García (en prensa) realizamos una caracterización de la mitigación cortés del desacuerdo, centrada en los contextos no conflictivos ( 3 a y $3 b$ ), algunos de cuyos resultados traemos aquí muy sucintamente, pues nos servirán de referencia para el análisis de la potenciación descortés del desacuerdo en el contexto de tensión (4). Señalemos, en primer lugar, que las estrategias de mitigación cortés aisladas fueron las siguientes:

\subsection{DiRIGIDAS A LA IMAGEN CUALITATIVA:}

1. Mostrar respeto por la opinión del interlocutor.

2. Minimizar el desacuerdo.

\footnotetext{
${ }^{2}$ El porcentaje de hablantes que se muestra dispuesto a expresar su desacuerdo en el contexto informal distendido de 3a es un $91,67 \%$ en los españoles y un 73,33\% en los ingleses; sin embargo, en el contexto conflictivo de 4, opta por la expresión del desacuerdo solo un $50 \%$ de los españoles y un $34,17 \%$ de los ingleses (Fernández García y Aguayo Cruz, 2019).

${ }^{3}$ Somos plenamente conscientes de que, como muy bien explica Briz (2012: 34), la cortesía (actividad social) y la atenuación (estrategia lingüística) son solo una "pareja de conveniencia", al igual que lo son descortesía y la intensificación. Es decir, que el vínculo entre ellas no tiene por qué darse necesariamente de ese modo, resultando la clave la configuración del acto comunicativo en que se manifiestan. Por ello acotamos la validez de nuestra afirmación a "lo relativo a los contextos descritos".
} 


\subsection{DIRIGIDAS A LOS DERECHOS DE EQUIDAD:}

3. Relativizar el peso de la propia opinión, restarle rotundidad.

4. Invitar a reconsiderar.

\subsection{DIRIGIDAS A LOS DERECHOS AFILIATIVOS:}

5. Lamentar el desacuerdo o disculparse por él.

6. Mostrar que el desacuerdo es inevitable o incompatible con los deseos de franqueza.

7. Mostrar camaradería o buen humor.

Y añadamos que, conforme al estudio cuantitativo realizado, la distribución de uso de los grupos de estrategias fue la siguiente (Fernández García, en prensa):

Tabla 2. Uso de estrategias de mitigación según el aspecto social al que se orientan

\begin{tabular}{|c|c|c|c|c|c|c|}
\cline { 2 - 7 } \multicolumn{1}{c|}{} & \multicolumn{3}{c|}{ Pregunta 3a } & \multicolumn{3}{c|}{ Pregunta 3b } \\
\cline { 2 - 7 } \multicolumn{1}{c|}{} & $\begin{array}{c}\text { Imagen } \\
\text { cualitativa }\end{array}$ & $\begin{array}{c}\text { Derechos } \\
\text { de equidad }\end{array}$ & $\begin{array}{c}\text { Derechos } \\
\text { afiliativos }\end{array}$ & $\begin{array}{c}\text { Imagen } \\
\text { cualitativa }\end{array}$ & $\begin{array}{c}\text { Derechos } \\
\text { de equidad }\end{array}$ & $\begin{array}{c}\text { Derechos } \\
\text { afiliativos }\end{array}$ \\
\hline Jaén & $22,35 \%$ & $61,18 \%$ & $14,12 \%$ & $23,08 \%$ & $55,77 \%$ & $21,15 \%$ \\
\hline Coventry & $26,47 \%$ & $47,06 \%$ & $24,99 \%$ & $00,00 \%$ & $59,26 \%$ & $37,03 \%$ \\
\hline
\end{tabular}

Sin entrar ahora en más detalles, dejemos constancia de cómo, a la hora de mitigar, tanto los informantes españoles como los ingleses hicieron un uso mayoritario de las estrategias dirigidas a los derechos de equidad, siendo ello así tanto en el contexto informal distendido de 3 a como en el contexto formal asimétrico de $3 \mathrm{~b}$.

Cerramos este apartado añadiendo que, en Fernández García y Aguayo Cruz (2019), cuando estudiábamos la decisión que, en los diferentes contextos analizados, adoptaron nuestros informantes entre exteriorizar o no su desacuerdo, aislamos también una nómina de reacciones alternativas/complementarias al desacuerdo. Fueron las siguientes:

1. Asumir con naturalidad el desacuerdo.

2. Reclamar respeto para la propia opinión; criticar o lamentar la actitud del interlocutor.

3. Pedir explicaciones adicionales.

4. Dar la razón al interlocutor, aceptar su opinión.

5. Callarse, dejarlo pasar, no hacer caso.

6. Poner fin a la conversación o cambiar de tema.

7. Pedir segunda opinión.

8. Seguir la corriente.

9. Mostrar un enfrentamiento abierto.

10. Buscar un escenario de mayor calma.

Como veremos, a la hora de estudiar las estrategias de potenciación del desacuerdo, resulta interesante en ocasiones observar con qué otras reacciones aparece combinado su uso. 


\section{LA POTENCIACIÓN DESCORTÉS DEL DESACUERDO}

Tal y como ha quedado explicado en $\S 3$, nuestro análisis de la potenciación descortés del desacuerdo parte de los datos obtenidos en la pregunta 4 (marco informal de tensión), puesto que, al contrario de lo que ocurría en 3a (marco informal distendido) y $3 \mathrm{~b}$ (marco formal asimétrico), es en ella en la que el porcentaje de casos de potenciación resulta abrumadoramente mayoritario. En cuanto al marco situacional de la pregunta 4, recordemos que la conversación amistosa de 3 a se transforma en una discusión, cambio que hace transformarse las estructuras conversacionales, convirtiendo en estructuralmente preferida la expresión del desacuerdo, cuya potenciación, orientada a intensificar la asertividad de la enunciación, adquiere tintes clara y sistemáticamente descorteses.

\subsection{ANÁLISIS CUALITATIVO: CATEGORÍAS ANALÍTICAS AISLADAS}

El análisis cualitativo de las respuestas de nuestros informantes a la pregunta 4 ha revelado que la potenciación descortés del desacuerdo se ejecuta de manera recurrente mediante seis estrategias (que, aunque no guardan un paralelismo sistemático con las estrategias de mitigación que presentábamos en $\S 3$, constituyen, en buena medida, su reverso). Las presentamos agrupadas según el aspecto social al que se orientan, conforme a las categorías del rapport management de Spencer-Oatey $(2002,2008)$ :

\subsubsection{Dirigidas a la imagen cualitativa: ${ }^{4}$}

1. Criticar bruscamente o descalificar la postura del interlocutor. Frente a las estrategias mitigadoras que suponían un modo de poner en valor la opinión del interlocutor, el hablante pone el énfasis contra la postura expresada por aquel, criticándola con dureza o llegando, incluso, a descalificarla.

2. Devolverle la crítica al interlocutor. Podría considerarse un subtipo específico de la estrategia anterior, en el que la crítica y la descalificación se materializan en un el que no tiene ni idea eres tú o alguna fórmula semejante.

3. Maximizar el desacuerdo. Frente a los propósitos mitigadores de restar importancia al desacuerdo, de acotarlo, el hablante se propone aquí amplificarlo, hacer patente que no hay puntos de unión entre ambas posturas, acción que implica - como las estrategias anteriores - una evaluación negativa de las cualidades del interlocutor.

\subsubsection{Dirigidas a los derechos de equidad:}

4. Mostrar firmeza y rotundidad en la opinión. Frente al deseo mitigador de relativizar el peso de la propia opinión para no mostrarse impositivo, el hablante busca aquí justo lo contrario, a saber, mostrarse firme y rotundo en su postura, resultar manifiestamente impositivo.

\footnotetext{
${ }^{4}$ Como puede observarse, dentro de la imagen, solo hemos aislado el uso de estrategias dirigidas a la imagen cualitativa, pero no a la imagen social ni a la relacional.
} 


\subsubsection{Dirigidas a los derechos afiliativos:}

5. Mostrar enfado. Frente a posturas conciliadoras que buscaban algún tipo de implicación emocional con el interlocutor, el hablante deja ver con claridad su enfado y la ausencia, por consiguiente, de dicha implicación.

6. Mostrar incredulidad. Como en el caso anterior, lejos de intentar tender puentes afectivos con el interlocutor, el hablante muestra su perplejidad ante la actitud adoptada por este, haciendo ver que la considera por completo fuera de lugar.

Paralelamente a dicha nómina de estrategias funcionales, el análisis cualitativo de las respuestas de nuestros informantes nos ha permitido aislar los mecanismos lingüísticodiscursivos utilizados de forma recurrente para implementarlas. Son estos:

1. Uso de elementos nominales o adjetivales extremos. Mecanismo ligado a la estrategia 1. Se concreta en elementos como disparate o estupidez, descabellado o radical.

2. Uso de expresiones adverbiales intensificadoras. Mecanismo ligado principalmente a la estrategia 3. Se concreta en expresiones como strongly, completely, at all, para nada, en absoluto.

3. Uso de construcciones enfatizadoras de la contradicción. Mecanismo ligado a la estrategia 4. Se concreta en construcciones como of course, I know, I do know, no way.

4. Uso de expresiones beligerantes. Mecanismo ligado principalmente a la estrategia 2. Se concreta sobre todo en variaciones de la fórmula el que no tiene ni idea eres tú e it is you who doesn't know what you are talking about, además de otras como lo que me dé la gana o no te lo crees ni tú.

5. Uso de expresiones malsonantes. Mecanismo ligado principalmente a la estrategia 5. Se concreta en expresiones como esa mierda, cojones, ni puta idea.

6. Uso de exclamaciones y vehemencia en la elocución; elevación de la voz. Mecanismo ligado principalmente a las estrategias 4, 5 y 6 . Se manifiesta de muy variadas formas, como, por ejemplo, iqué dices! u oh, my God!.

7. Uso de interrogaciones retóricas. Mecanismo ligado principalmente a las estrategias 1, 2, 4 y 6. Aparece en variadas manifestaciones, como, por ejemplo, ¿y tú sí? o how dare you?.

8. Uso de ironías. Mecanismo ligado principalmente a la estrategia 2. Se concreta fórmulas como y tú tienes la verdad absoluta... o and you think you know...

9. Expresión explícita de incredulidad. Mecanismo ligado a la estrategia 6. Se concreta en fórmulas del tipo no me lo puedo creer o I can't believe you have said that.

\subsection{ANÁLISIS CUANTITATIVO: DATOS GENERALES}

A partir de las categorías de análisis ahora descritas, el primer paso en la caracterización del uso de la potenciación descortés por parte de nuestros informantes será la puesta en consideración de los datos generales sobre el aspecto social del destinatario contra el que se dirige dicha descortesía. Aparecen en la tabla 3: 
Tabla 3. Uso de estrategias de potenciación según el aspecto social al que se orientan

\begin{tabular}{|c|c|c|c|}
\cline { 2 - 4 } \multicolumn{1}{c|}{} & $\begin{array}{c}\text { Imagen } \\
\text { cualitativa }\end{array}$ & $\begin{array}{c}\text { Derechos } \\
\text { de equidad }\end{array}$ & $\begin{array}{c}\text { Derechos } \\
\text { afiliativos }\end{array}$ \\
\hline Jaén & $64,10 \%$ & $12,82 \%$ & $23,08 \%$ \\
\hline Coventry & $42,86 \%$ & $23,81 \%$ & $33,33 \%$ \\
\hline
\end{tabular}

Estas cifras globales revelan ya hechos de gran interés, tanto por sí mismas cuanto en comparación con las relativas al uso de las estrategias de mitigación en 3a (contexto informal distendido) y $3 \mathrm{~b}$ (contexto formal asimétrico), que mostrábamos en la tabla 2 (véase $\S 3$ ). Particularmente llamativo resulta el hecho de que, mientras que allí, hablando de mitigación, sobresalían de manera sostenida (en ambas culturas y en ambos contextos) las estrategias dirigidas a los derechos de equidad, aquí son las orientadas a la imagen cualitativa las que adquieren un neto protagonismo.

De este modo, la tónica general a la hora de mitigar la formulación del desacuerdo era orientarla hacia la idea de la no imposición sobre el interlocutor, con muestras de respeto hacia su autonomía, ya fuera relativizando el peso de la propia opinión o restándole rotundidad (estrategia 3), ya fuera invitándole a reconsiderar (estrategia 4) en lugar de intentar imponerle el propio criterio. A la hora de intensificar la fuerza del desacuerdo, sin embargo, hallamos un nítido protagonismo de las estrategias dirigidas contra la imagen cualitativa. Ello supone que, cuando lo que se propone el hablante es atacar a su interlocutor, intensificando la vertiente conflictiva y descortés de la comunicación, el objetivo del ataque tiende a ser el componente afectivo, el deseo de sentirse valorado positivamente por los demás. Debemos incidir, además, en el hecho de que dicho cambio en la estrategia comunicativa frente al desacuerdo, siendo común a los hablantes españoles e ingleses, adquiere una fuerza particularmente marcada en los españoles, en los que el porcentaje de ataques a la imagen cualitativa $(64,10 \%)$ es un $50 \%$ superior que en los ingleses $(42,86 \%)$.

\subsection{DATOS ESPECÍFICOS Y ANÁLISIS DE EJEMPLOS}

Pasemos ahora al análisis de las distintas estrategias utilizadas, junto con los mecanismos característicos en los que se materializan. Conforme a lo explicado en $\S 4.1$, recordemos, en primer lugar, que son tres las estrategias de potenciación dirigidas contra la imagen cualitativa del interlocutor. Frente a la estrategia 1 de mitigación (mostrar respeto por la opinión del interlocutor), ${ }^{5}$ encontrábamos la estrategia 1 de potenciación (criticar bruscamente o descalificar la postura del interlocutor) y su variante, la estrategia 2 de potenciación (devolverle la crítica al interlocutor); $\mathrm{y}$, frente a la estrategia 2 de mitigación (minimizar el desacuerdo), encontrábamos la estrategia 3 de potenciación (maximizar el desacuerdo).

En la estrategia 1 de potenciación, por tanto, frente a la idea de poner en valor la opinión del interlocutor, el hablante se sitúa en una posición diametralmente opuesta, la de atacarla; y en la 2 dicho ataque cobra la forma específica de devolverle el ataque con un - con variaciones - el que no tiene ni idea eres tú. Pues bien, tal descalificación con tintes personales, concretamente la de la estrategia 2, es la que se sitúa en primer lugar tanto entre los informantes españoles como entre los ingleses a la hora de potenciar la fuerza de su desacuerdo. Lo vemos en la tabla 4:

\footnotetext{
${ }^{5}$ Véase en $§ 3$ la nómina completa de estrategias de mitigación.
} 
Tabla 4. Estrategias de potenciación, ordenadas conforme a su importancia porcentual en cada nacionalidad ${ }^{6}$

\begin{tabular}{|c|c|c|c|}
\hline \multicolumn{2}{|l|}{ JAÉN } & \multicolumn{2}{|l|}{ COVENTRY } \\
\hline Estrategia & $\%$ & Estrategia & $\%$ \\
\hline $\begin{array}{l}\text { Devolverle la crítica al } \\
\text { interlocutor }\end{array}$ & 46,15 & $\begin{array}{l}\text { Devolverle la crítica al } \\
\text { interlocutor }\end{array}$ & 23,81 \\
\hline $\begin{array}{l}\text { Criticar bruscamente o } \\
\text { descalificar la postura } \\
\text { del interlocutor }\end{array}$ & 17,95 & $\begin{array}{l}\text { Mostrar firmeza y } \\
\text { rotundidad en la opinión }\end{array}$ & 23,81 \\
\hline $\begin{array}{l}\text { Mostrar firmeza y } \\
\text { rotundidad en la opinión }\end{array}$ & 12,82 & Mostrar incredulidad & 23,81 \\
\hline Mostrar incredulidad & 12,82 & $\begin{array}{l}\text { Criticar bruscamente o } \\
\text { descalificar la postura } \\
\text { del interlocutor }\end{array}$ & 14,29 \\
\hline Mostrar enfado & 10,26 & Mostrar enfado & 9,52 \\
\hline $\begin{array}{l}\text { Maximizar el } \\
\text { desacuerdo }\end{array}$ & -- & $\begin{array}{l}\text { Maximizar el } \\
\text { desacuerdo }\end{array}$ & 4,76 \\
\hline
\end{tabular}

Eso sí, siendo cierto que la estrategia 2 (devolverle la crítica al interlocutor) se sitúa en primer lugar en ambos casos, los datos muestran con claridad su distinto protagonismo entre españoles y entre ingleses: su peso en los primeros prácticamente dobla al que tiene en los segundos (46,15\% frente a 23,81\%); y, además, mientras que en el caso español el porcentaje de tal estrategia multiplica por 2,5 el de la siguiente (estrategia 1, criticar bruscamente o descalificar la postura del interlocutor, con un 17,95\%), en los ingleses tiene una importancia porcentual idéntica a la de otras dos estrategias.

Redondeado, pues, casi una de cada dos veces que el informante español se decide a potenciar su desacuerdo en ese contexto de tensión en que su interlocutor le ha acusado de no tener ni idea de lo que habla, lo hace devolviéndole la crítica. Tal acción se plasma con frecuencia en el mecanismo del uso de expresiones beligerantes, que se presenta, por ello, como el mecanismo de potenciación más frecuente entre los españoles en términos absolutos. Vemos un ejemplo en (1):

(1) Tú no sabes si yo tengo contacto de primera mano, y si no lo tuviera puedo opinar lo que me dé la gana. A lo mejor el que no tiene ni idea eres tú, que estás todo el rato negando lo que digo, como si fueses la única persona que tiene razón en el mundo, y no $(\mathrm{J} 2)^{7}$

Podemos apreciar cómo este informante de Jaén se sirve por partida doble del mecanismo del uso de expresiones beligerantes, para devolverle la crítica al interlocutor - estrategia 2- ("a lo mejor el que no tiene ni idea eres tú") y para replicar con dureza a su postura - estrategia 1- ("puedo opinar lo que me dé la gana").

\footnotetext{
${ }^{6}$ En los casos en que los porcentajes son idénticos, el orden en que aparecen las estrategias en la tabla sigue el del listado en que fueron presentadas en $\S 4.1$.

${ }^{7}$ Los informantes de Jaén y Coventry son identificados mediante J o C junto con el número de cuestionario. Por otra parte, el texto aparece entrecomillado cuando es presentado por el informante como una representación literal de lo que diría en esa situación, mientras que aparece sin comillas cuando corresponde a texto explicativo.
} 
Junto a las expresiones beligerantes, también son relevantes en la ejecución de la estrategia 2 las ironías (ligadas principalmente a él en nuestros datos, pero de importancia global secundaria) y las interrogaciones retóricas (frecuentes aquí, pero también en otras estrategias, y segundo mecanismo de potenciación más frecuente entre los españoles, detrás del uso de expresiones beligerantes). ${ }^{8}$ Vemos sendos ejemplos en (2) y (3):

(2) Sííi... Clarooo. Y tú tienes la verdad absoluta, sin lugar a dudas... Anda ya y vete un poquito lejos (J73).

(3) Reaccionaría haciéndole sentir a él más molesto aún. Mis palabras: «¿Que no sé lo que estoy hablando! ¿Y tú sí? ¡No tienes ni idea! Hablar contigo es igual que hablar con la pared» $(\mathrm{J} 112)$.

En (2), con una doble ironía, el informante devuelve la crítica al interlocutor - estrategia 2-(y tú tienes la verdad absoluta, sin lugar a dudas), además de haber descalificado previamente su postura - estrategia 1-(Síí... Clarooo); y, para rematar la dura réplica, se sirve de la reacción complementaria 9, mostrar un enfrentamiento abierto (anda y vete un poquito lejos). ${ }^{9}$ La dura réplica de (3), por su parte, además de echar mano de nuevo de la reacción complementaria 9 (Hablar contigo es igual que hablar con la pared), utiliza, entre otros mecanismos, sendas interrogaciones retóricas para descalificar la postura del interlocutor - estrategia 1 - (¿Que no tengo ni idea!) y para devolverle la crítica - estrategia 2- (¿Y tú sí?).

En el caso de los hablantes ingleses, como decíamos, la estrategia 2 funciona de manera semejante a los españoles y se encuentra también entre las más importantes (compartiendo la primera posición con otras dos), pero lejos del protagonismo que adquiere en estos. Se plasma esencialmente en el uso de expresiones beligerantes - por ejemplo, en (4) — y en algún caso aislado también en forma irónica — como en (5)—: ${ }^{10}$

(4) My friend, it is you who doesn't know what you are talking about. I think we may just have to agree to disagree $(\mathrm{C} 27)$.

(5) Oh, that's nonsense. That's so arrogant. And you think you know what you're talking about... (C67).

Más allá de la ironía de (5), combinada con la reacción complementaria 2 (reclamar respeto para la propia opinión; criticar o lamentar la actitud del interlocutor), llama la atención, en (4), que, aun a pesar de la dureza que intrínsecamente lleva aparejado el uso de la estrategia 2, esta se suaviza con el vocativo inicial, y luego, además, la intervención se remata con la reacción complementaria 1 (asumir con naturalidad el desacuerdo), de perfil nítidamente conciliador: se trata de una actitud muy inglesa, y bastante extraña entre españoles.

\footnotetext{
${ }^{8}$ No podemos detenernos aquí en la revisión teórica de los complejos fenómenos de la ironía y la interrogación retórica. Nos remitimos, por ello, a la concepción manejada y desarrollada por extenso en estudios anteriores, ligados específicamente al análisis de la comunicación política: Fernández García (2001) y (2017: 198-201) en el caso de la ironía, y Fernández García (2002) y (2017: 192-198) en el de la interrogación retórica.

9 Conforme a lo explicado en $\S 3$, nos referimos a la nómina de otras reacciones alternativas o complementarias al desacuerdo aisladas en Fernández García y Aguayo Cruz (2019).

${ }^{10}$ A diferencia del caso de los españoles, no aparecen aquí interrogaciones retóricas. Hemos de señalar que la interrogación retórica es el mecanismo más habitual entre los ingleses a la hora de ejecutar estrategias de potenciación, pero aparece ligado a otras, como veremos.
} 
El enorme peso de las estrategias dirigidas contra la imagen en los hablantes españoles (recordemos, conforme a la tabla 3, que las utilizan en el $64,10 \%$ de las ocasiones en que potencian el desacuerdo) tenía que ver, sobre todo, con la gran importancia de esta estrategia 2, pero también con el hecho de que - aunque a mucha distancia - la segunda estrategia más usada es la 1 (criticar bruscamente o descalificar la postura del interlocutor), también orientada a la imagen, que entre los ingleses se encuentra en cuarta posición (tabla 4). Según señalábamos más arriba, frente al propósito mitigador de poner en valor la opinión del interlocutor como forma de evaluación positiva de sus cualidades personales (estrategia 1 de mitigación: mostrar respeto por la opinión del interlocutor), aquí se pone el énfasis precisamente contra dicha postura, llegado en ocasiones a descalificarla. Sus mecanismos característicos de ejecución son, por un lado, el uso de elementos nominales o adjetivales extremos (especialmente ligado a esta estrategia), y por otro, de nuevo el uso de interrogaciones retóricas. Vemos sendos ejemplos en (6) y (7):

(6) ¿Realmente piensas que no tengo ni idea de lo que estás hablando? ¿Yo? Tú eres el que realmente no tiene ni puta idea, ya que tus argumentos son estupideces (J84).

(7) Vete a la mierda. Tú qué sabes lo que yo sé o dejo de saber (J19).

Vemos en (6) que la estrategia 1 (criticar bruscamente o descalificar la postura del interlocutor) se materializa en el uso de un elemento nominal extremo (tus argumentos son estupideces), como cierre de una airada intervención en la que se acumulan también la estrategia 2 (devolverle la crítica al interlocutor), combinando el uso de expresiones beligerantes con el uso de expresiones malsonantes (Tú eres el que realmente no tiene ni puta idea), y la estrategia 6 (mostrar incredulidad), implementada mediante una interrogación retórica (¿Realmente piensas que no tengo ni idea de lo que estás hablando?).

En cuanto al ejemplo (7), encontramos una nueva reacción virulenta de un informante español, esta vez, como anunciábamos, mediante una interrogación retórica para ejecutar la estrategia 1 (Tú qué sabes lo que yo sé o dejo de saber), después de haber hecho uso de la reacción alternativa 9 (mostrar un enfrentamiento abierto: Vete a la mierda). Y dicho mecanismo, el de la interrogación retórica, es precisamente el que predomina en los informantes ingleses para ejecutar esta estrategia 1, que entre ellos recordemos - se sitúa en cuarta posición. Es el caso, por ejemplo, de (8), donde, como ocurre con frecuencia, aunque se busque igualmente potenciar el desacuerdo, el tono general se muestra más suave que en muchas de las intervenciones de los informantes españoles:

(8) Question their valitidy/where is their knowledge coming from: "What makes you think you know so much about this?" (C109).

Para cerrar las consideraciones relativas a las estrategias de potenciación ligadas a la imagen, nos referimos brevemente a la estrategia 3, maximizar el desacuerdo. Como puede observarse en la tabla 4, se trata de una estrategia de importancia marginal, dado que no es utilizada por los informantes españoles y se sitúa en último lugar entre los ingleses. Aclaremos, no obstante, que tiene cierta presencia tanto en unos como en otros en las contadas ocasiones en que aparecen desacuerdos potenciados en los contextos $3 \mathrm{a} o$ 
3b. ${ }^{11}$ De hecho, dentro del escaso espacio que los desacuerdos potenciados ocupan en tales contextos, resulta una de las estrategias más relevantes, pero, cuando el ambiente se vuelve tenso, en el caso del contexto 4, los hablantes parecen optar por otras estrategias de mayor fuerza confrontacional. Su plasmación característica es el uso de expresiones adverbiales intensificadoras:

(9) I would say that they have a right to their opinion, but I strongly disagree and I feel upset that they don't respect my opinion (C36).

Vemos cómo la expresión explícita del desacuerdo (disagree), lejos de ser minimizada de algún modo, es abiertamente maximizada (strongly), acción que el informante combina con el uso de la reacción complementaria 2 (reclamar respeto para la propia opinión; criticar o lamentar la actitud del interlocutor).

Señalábamos más arriba que el protagonismo de los ataques potenciados contra la imagen cualitativa mostraba la preferencia de los hablantes, a la hora de inclinarse hacia la vertiente conflictiva del diálogo, por el componente afectivo, el deseo de sentirse valorado positivamente por los demás. $\mathrm{Y}$ hemos de insistir en que, siendo común a españoles e ingleses esta preferencia por los ataques contra la imagen cualitativa, son los primeros los que sobresalen ampliamente en dicho sentido. En contrapartida, mientras que los derechos de equidad — vinculados a la idea de la no imposición- eran el elemento nuclear en torno al que giraban las acciones mitigadoras, quedan relegados a un papel secundario en la vertiente atacante. Solo se manifiestan, de hecho, en una estrategia, la 4 (mostrar firmeza y rotundidad en la opinión), que viene a ser el reverso de la estrategia 3 de mitigación (relativizar el peso de la propia opinión, restarle rotundidad). El propósito funcional de tal estrategia de potenciación en el contexto de una discusión es claro: mostrarse firme y rotundo precisamente con la intención de resultar manifiestamente impositivo ante el interlocutor.

Siendo claro el menor peso de los derechos de equidad en la acción potenciadora del desacuerdo frente a su papel en la vertiente mitigadora, hemos de diferenciar, no obstante, entre la actitud de españoles e ingleses en este sentido: en los primeros esta estrategia 4 se sitúa en tercer lugar, con un $12,82 \%$, mientras que en los segundos, con un $23,81 \%$, comparte la primera posición con la estrategia 2 (devolverle la crítica al interlocutor), ya tratada, y la 6 (mostrar incredulidad), de la que trataremos más adelante. Dicha diferencia se relaciona con lo que podríamos entender, conforme a los datos de la tabla 3, como una actitud más equilibrada del hablante inglés en relación con el objetivo social al que dirige la potenciación descortés de sus desacuerdos (que se reparte de manera más homogénea entre la imagen cualitativa, los derechos de equidad y los derechos afiliativos), frente al abrumador protagonismo que la potenciación orientada hacia la imagen cualitativa tiene en el hablante español.

En lo tocante a los mecanismos mediante los que dicha estrategia se implementa, en el caso de los españoles tiende a manifestarse en forma de interrogación retórica (mecanismo a cuya multifuncionalidad ya hemos hecho referencia), como muestra el ejemplo (10); por su parte, los ingleses, tendentes a la interrogación retórica para otras funciones, optan aquí por el uso de construcciones enfatizadoras de la contradicción,

\footnotetext{
${ }^{11}$ Recordemos que, según explicábamos en $\S 2$, el contexto 3a correspondía a la conversación informal con el amigo (esto es, el marco informal distendido) y el contexto $3 b$, a la conversación con el médico (esto es, el marco formal asimétrico). $\mathrm{Y}$ en $\S 3$ señalábamos que, puesto que en ambos marcos situacionales la expresión del desacuerdo estaba ligada mayoritariamente a la mitigación cortés, nuestro análisis de la potenciación descortés se centra en el contexto 4 (esto es, en la conversación con el amigo, convertida ahora en discusión).
} 
mecanismo característicamente ligado a esta estrategia, que vemos ejemplificado en (11); en ambas culturas se manifiesta, además, en el uso de exclamaciones y vehemencia en la elocución, como vemos en el ejemplo inglés de (12):

(10) Ya me sentiría bastante incómodo y algunas palabras podrían ser: «Anda ya... ¿Que no tengo ni idea?» (J8).

(11) Might use phrases such as «what gives you the right to think that?» or «of course I know what I'm talking about» (C11).

(12) If I knew enough about the subject I would very loudly state the facts as I believed them. I might say something personal at this point. «You are an idiot», etc. (or worse). I would question their beliefs (C48).

Es interesante, en (10), cómo el informante refleja en sus palabras la incidencia del cambio contextual, con el paso del contexto distendido de 3 a al contexto de tensión de 4, circunstancia con la que parece justificar la decisión de potenciar el desacuerdo ( $\mathrm{Ya}$ me sentiría...); dicha potenciación, por otra parte, la ejecuta con esa interrogación retórica que podría parafrasearse como por supuesto que tengo idea, claro posicionamiento de firmeza y rotundidad. En (11), el informante inglés, tras descalificar la afirmación de su interlocutor (uso de la estrategia 1: criticar bruscamente o descalificar la postura del interlocutor) mediante una interrogación retórica, se sirve de ese of course I know, construcción enfatizadora de la contradicción, para ejecutar su muestra de firmeza y rotundidad (la decir, la estrategia 4, de la que ahora tratamos). En (12), por último, el informante inglés muestra su intención de subir ostensiblemente el tono de su elocución (I would very loudly state the facts), al tiempo que servirse de la estrategia complementaria 9 (mostrar un enfrentamiento abierto), descalificando a su interlocutor.

Detengámonos, por último, en los ataques vinculados a los derechos afiliativos. Las estrategias mitigadoras con ellos relacionadas (la 5, lamentar el desacuerdo o disculparse por él; la 6, mostrar que el desacuerdo es inevitable o incompatible con los deseos de franqueza; y la 7, mostrar camaradería o buen humor) estaban orientadas a mostrar respeto hacia el interlocutor y su papel social, así como cierta implicación emocional con él. Como reverso de dichos propósitos, encontramos aquí las estrategias de potenciación 5 (mostrar enfado) y 6 (mostrar incredulidad), que, lejos de intentar tender puentes afectivos, exhiben una ausencia de implicación emocional. El conjunto de las dos, es decir, el bloque de las estrategias de potenciación dirigidas a los derechos afiliativos, muestra un papel secundario en relación con la imagen, pero ligeramente superior al que tenía en el plano de la mitigación, y desde luego, superior al de los derechos de equidad, que — según ha quedado explicado más arriba — resulta escaso en las acciones de potenciación.

Por otro lado, en lo tocante a la comparación intercultural, el peso de las acciones dirigidas contra los derechos de equidad en el plano de la potenciación sigue siendo, como ocurría en la mitigación, mayor entre los ingleses que entre los españoles, y en una proporción semejante a la de allí (véanse tablas 2 y 3 ). Y, en cuanto al papel de cada una de las estrategias, las dos muestran un peso semejante - y secundario- entre los españoles (la 6, mostrar incredulidad, comparte el tercer puesto, con un 12,82\%; y la 5, mostrar enfado, se sitúa a continuación, con un 10,26\%). En el caso de los ingleses, sin embargo, su peso es muy diferente, de manera que la 6 sí que posee relevancia (siendo una de las tres que comparten la primera posición, con un $23,81 \%$ ), mientras que la 5 muestra una importancia menor (se queda en un 9,52\%). 
Aunque con diferencias entre unos y otros, por tanto, la más usada en este ámbito de los derechos de afiliación es la estrategia 6 (mostrar incredulidad). Con su uso, el hablante se muestra perplejo ante su interlocutor, haciéndole ver que considera de todo punto fuera de lugar lo que acaba de decir. En los hablantes de ambas nacionalidades se manifiesta en forma de interrogaciones retóricas — como en (13) - y de expresiones explícitas de incredulidad - como en (14)-; y, particularmente en el caso de los españoles, también mediante el uso de exclamaciones y vehemencia en la elocución como en (15)-:

(13) «¡Tú estás tonta! ¿De verdad me estás diciendo lo que me estás diciendo?». Y ya intentaría debatirle con argumentos (J52).

(14) This would be very unlikely! That is because I would not normally allow a discussion with a friend to became uncomfortable to this degree. If it did, I might say: «I can't believe you have said that... Why are you saying that?» (C43).

(15) ¡Pero qué dices!, ¡qué me estás contando! Eso no es así. Vamos a dejar el tema ya (J65).

En (13), la informante combina la expresión de incredulidad mediante una interrogación retórica con la reacción complementaria 9 (mostrar un enfrentamiento abierto), descalificando a su interlocutora. En (14), por su parte, encontramos un argumento que se repite con cierta frecuencia entre los informantes ingleses, a saber, el de que la conversación con un amigo raramente llegaría a un escenario como el descrito en la pregunta 4; llegado el caso, no obstante, la informante optaría por explicitar su incredulidad. En (15), por último, las muestras de incredulidad en forma de exclamaciones son rematadas con la reacción complementaria 6 (poner fin a la conversación o cambiar de tema).

En cuanto a la estrategia 5 (mostrar enfado), resulta una muestra manifiesta de la ausencia de implicación emocional con el interlocutor a causa de su actitud discursiva, como reverso de los intentos conciliadores de las estrategias de mitigación 5 (lamentar el desacuerdo o disculparse por él) y 7 (mostrar camaradería o buen humor). Más allá de algún caso de interrogación retórica, se manifiesta principalmente en el uso de exclamaciones y vehemencia en la elocución, y en el uso de expresiones malsonantes. La utilización conjunta de ambos mecanismos queda clara en el ejemplo inglés de (16), en el que, aunque no se reproduce discurso literal, la informante describe con detalle su hipotética reacción:

(16) My blood pressure would be raised, my face would get warm as I would be quite annoyed inside. I would raise my voice as I stressed to them the reasons for my opinion. [...] I would definitely swear at them too (C49).

\section{CONCLUSIONES}

Explicábamos al comienzo que, conforme a los avances previos de nuestra investigación, partíamos de la base de que nuestros informantes, cuando optan por llevar cabo una mitigación cortés del desacuerdo, lo hacen de manera mayoritaria sirviéndose de estrategias orientadas hacia la no imposición (esto es, hacia los derechos de equidad), cosa que ocurre de forma particularmente acentuada entre los españoles. A partir de ahí, el análisis desarrollado ha dejado claro que, cuando de lo que se trata es de la potenciación descortés del desacuerdo, es el deseo de sentirse valorado (esto es, la imagen cualitativa) 
el que se convierte en principal objetivo de las acciones verbales beligerantes, hecho que - de nuevo - ocurre de manera particularmente acentuada entre los hablantes españoles. Recordemos que tales acciones de potenciación vinculadas a la imagen alcanzan en los españoles el $64,10 \%$ del total, con dos de las estrategias dirigidas a la imagen ocupando los primeros lugares (especialmente la 2, devolverle la crítica al interlocutor, pero también la 1, criticar bruscamente o descalificar la postura del interlocutor) y a una significativa diferencia de las siguientes. En el caso de los informantes ingleses, en contrapartida, la actitud potenciadora del desacuerdo parece ser socialmente más equilibrada, en el sentido de que encontramos tres estrategias empatadas en primer lugar, y cada una de ellas dirigida a un aspecto social distinto (la 2, devolverle la crítica, vinculada a la imagen cualitativa; la 4, mostrar firmeza y rotundidad en la opinión, vinculada a los derechos de equidad; y la 6, mostrar incredulidad, vinculada a los derechos afiliativos).

Hemos comprobado, por otra parte, que el tono de los desacuerdos potenciados en los informantes ingleses tiende, en general, a ser más suave que en los españoles. Y en aquellos, además, adquiere mucho mayor protagonismo que en los españoles (prácticamente el doble) la única estrategia dirigida a los derechos de equidad, es decir, a la no imposición (la estrategia 4, mostrar firmeza y rotundidad en la opinión). No obstante, en conjunto, la potenciación orientada a los derechos de equidad, con solo una estrategia, resulta secundaria, por detrás - tanto en ingleses como en españoles - del bloque que conforman las dos estrategias dirigidas a los derechos afiliativos, que vienen a mostrar la ausencia de implicación emocional entre los interlocutores, ya sea exteriorizando enfado (estrategia 5), ya sea reflejando incredulidad (estrategia 6). De este modo, apreciamos que, en conjunto, la potenciación descortés del desacuerdo cobra un tinte afectivo o emocional en la inmensa mayoría de los casos, siendo muy limitadas las acciones potenciadoras dirigidas contra el deseo de no imposición. Ciertamente, la suma de las dirigidas a la imagen cualitativa y las orientadas a los derechos afiliativos alcanza el $87,18 \%$ en los informantes españoles y el $76,19 \%$ en los ingleses, aunque con una manifiesta diferencia del peso de tales componentes: nítido protagonismo de la imagen en el caso español, más equilibrado en el caso inglés. Y los derechos de equidad, como venimos diciendo, ocupan un papel secundario en conjunto, pero resultando apreciablemente más importantes en el caso de los hablantes ingleses.

Para concluir, respecto de nuestros objetivos específicos iniciales, estamos en condiciones de afirmar que:

a) la potenciación descortés del desacuerdo en un contexto de tensión desarrolla sus propios patrones, muy diferentes a los de la mitigación cortés en un contexto distendido;

b) pueden aislarse estrategias funcionales y mecanismos lingüístico-discursivos de uso recurrente para el cumplimiento de dicho objetivo discursivo; y

c) el uso de dichas estrategias y mecanismos dibuja perfiles apreciablemente diferenciados entre los hablantes españoles y los ingleses.

\section{REFERENCIAS BIBLIOGRÁFICAS}

Bousfield, D. 2008. Impoliteness in Interaction. Ámsterdam: John Benjamins.

Bravo, D. 1999. «¿Imagen positiva vs. imagen negativa? Pragmática socio-cultural y componentes de face». Oralia, 2: 155-184.

- D. 2003. «Actividades de cortesía, imagen social y contextos socioculturales: una introducción». En Primer Coloquio del Programa EDICE. La perspectiva no 
etnocentrista de la cortesia: identidad sociocultural de las comunidades hispanohablantes, ed. D. Bravo. Estocolmo: Universidad de Estocolmo, pp. 98108.

Briz, A. 2012. «La (no)atenuación y la (des)cortesía, lo lingüístico y lo social: ¿son pareja?». En Miradas multidisciplinares a los fenómenos de cortesía y descortesía en el mundo hispánico, eds. J. Escamilla Morales y G. Henry Vega. BarranquillaEstocolmo: Universidad del Atlántico-Programa Edice, pp. 33-75.

Brown, P., y S. C. Levinson. 1978. «Universals in language usage: politeness phenomena». En Questions and Politeness: Strategies in Social Interaction, ed. E. N. Goody. Cambridge: Cambridge University Press, pp. 56-311.

- 1987. Politeness. Some Universals in Language Use. Cambridge: Cambridge University Press.

Culpeper, J. 1996. «Towards an anatomy of impoliteness». Journal of Pragmatics, 25: 349-367.

—. 2011. Impoliteness. Using Language to Cause Offence. Cambridge: Cambridge University Press.

Fernández García, F. 2001. «Ironía y (des)cortesía». Oralia, 4: 103-127.

—. 2002. «Sobre la funcionalidad de la interrogación retórica en la oratoria políticoelectoral». En IV Congreso de Lingüistica General (vol. III), eds. M. ${ }^{a}$ D. Muñoz Núñez et alii. Cádiz: Universidad de Cádiz, pp. 989-998.

—. 2016a. «Hacia un análisis de la variación cultural, social y situacional en la percepción de la (des)cortesía». En Oralidad y análisis del discurso, eds. A. M. Bañón Hernández, M. M. Espejo Muriel, B. Herrero Muñoz-Cobo y J. L. López Cruces. Almería: Universidad de Almería, pp. 223-238.

-.2016b. «Bases teóricas para un estudio transcultural y variacionista de la (des)cortesía». Estudios de Lingüistica. Universidad de Alicante, 30: 79-100.

—. 2017. La descortesía en el debate electoral cara a cara. Sevilla: Editorial Universidad de Sevilla.

—. en evaluación. «Desacuerdo y (des)cortesía: un análisis de variación pragmática». Spanish in Context.

—. en prensa. «Estrategias de mitigación en la formulación del desacuerdo: estudio intercultural». En (Des)cortesía, actividades de imagen e identidad, eds. M. González Sanz, C. Fuentes Rodríguez y E. Brenes Peña. Sevilla: Editorial Universidad de Sevilla.

Fernández García, F., y M. ${ }^{a}$ D. Aguayo Cruz. 2019. «Variación cultural y situacional en la gestión del desacuerdo». Pragmalingüistica, 27: 10-31.

Fernández García, F., y T. Ortiz Viso. 2018. «Tres dimensiones en el análisis de la variación de la (des)cortesía». Revista de Investigación Lingüística, 21: 133-158.

Kotthoff, H. 1993. «Disagreement and concession in disputes: On the context sensitivity of preference structures». Language in Society, 22: 193-216.

Levinson, S. C. 1983. Pragmatics. Cambridge: Cambridge University Press.

Locher, M. A., y R. J. Watts. 2005. «Politeness Theory and Relational Work». Journal of Politeness Research, 1: 9-33.

Schneider, K. P. 2012. «Appropriate behaviour across varieties of English». Journal of Pragmatics, 44: 1022-1037. 
Spencer-Oatey, H. 2002. «Managing rapport in talk: Using rapport sensitive incidents to explore the motivational concerns underlying the management of relations». Journal of Pragmatics, 34: 529-545.

-. 2005. «(Im)Politeness, face and perceptions of rapport: unpackaging their bases and interrelationships». Journal of Politeness Research, 1: 95-119.

- 2007. «Theories of identity and the analysis of face». Journal of Pragmatics, 39: 639656.

-. 2008. «Face, (Im)Politeness and Rapport». En Culturally Speaking. Culture, Communication and Politeness Theory, ed. H. Spencer-Oatey. Londres: Continuum, pp. 11-47.

—., ed. 2008. Culturally Speaking. Culture, Communication and Politeness Theory (2. edición). Londres: Continuum.

Staley, L. 2018. Socioeconomic Pragmatic Variation. Ámsterdam: John Benjamins.

Watts, R. J. 2003. Politeness. Cambridge: Cambridge University Press.

Wierzbicka, A. 2003. Cross-Cultural Pragmatics. The Semantics of Human Interaction (2. ${ }^{a}$ ed.). Berlín: Mouton de Gruyter. 